\title{
Synthesis and Characterization of Acrylamide-Based Anionic Copolymer and Investigation of Solution Properties
}

\author{
H. Jamshidi and A. Rabiee \\ Iran Polymer and Petrochemical Institute, P.O. Box 14965/115, Tehran, Iran \\ Correspondence should be addressed to A. Rabiee; a.rabbii@ippi.ac.ir
}

Received 11 January 2014; Revised 17 June 2014; Accepted 17 June 2014; Published 21 July 2014

Academic Editor: Luigi Nicolais

Copyright ( 2014 H. Jamshidi and A. Rabiee. This is an open access article distributed under the Creative Commons Attribution License, which permits unrestricted use, distribution, and reproduction in any medium, provided the original work is properly cited.

The copolymer of acrylamide (AM) and 2-acrylamido-2-methyl-1-propane sulfonic acid (AMPS) was synthesized through radical solution polymerization by potassium persulfate as initiator. By changing the AMPS feed ratio from 10 to $70 \%$, and keeping other reaction conditions constant, different copolymers were synthesized. The techniques of Fourier transform infrared (FTIR) and nuclear magnetic resonance $\left({ }^{1} \mathrm{H}-{ }^{13} \mathrm{C}-\mathrm{NMR}\right)$ spectroscopy were used for identification of functional groups and confirmation of copolymers' structure. Intrinsic and apparent viscosity of samples were measured in aqueous sodium chloride solution under standard conditions. The anionic degree of copolymers was determined by back titration method and by ${ }^{13} \mathrm{C}-\mathrm{NMR}$ spectroscopy. Molecular weight of copolymers was determined by the Mark-Houwink relationship. The measured molecular weight of samples showed that we have acquired a high molecular weight product. The effect of different range of shear rates on solution viscosity was evaluated. The copolymer solutions showed non-Newtonian shear thinning behavior. The performance of copolymers with respect to shear resistance and molecular weight was evaluated from industry application standpoint.

\section{Introduction}

In recent years, special attention has been devoted to water soluble polymers. The most important requirements for technological applications of these polymers, such as high water solubility, easy and cheap synthetic route, shear resistance behavior, and chemical stability of the polymer structure, stand out plausibly. Among water-soluble polymers, acrylamide based copolymers and homopolymers provide a wide range of functionalities and benefits to a variety of applications [1].

Acrylamide based polymers are often used as thickeners, stabilizers, film formers, rheology modifiers, emulsifiers, lubricity aids, conditioners, and viscosity control agents for enhanced oil recovery $[2,3]$. Most applications of these polymers arise from their properties in solution, especially for their ability to modify the rheology of an aqueous medium. Polymer molecules would increase the viscosity as a result of their hydrodynamic volume, and viscosity may be further enhanced through intermolecular association [1]. The ideal polymer candidates for mobility control agents should have long-term thermal stability under the reservoir conditions of temperature and salinity. Moreover, they should be costeffective [2].

Many synthetic water soluble polymers have been tested for, but most studies have focused on polyacrylamide- (PAM) based polymers due to their availability and relatively low cost. The best mobility control agents in surfactant-polymer and polymer-augmented water flooding processes are conventional polymers such as polyacrylamides, especially partially hydrolyzed polyacrylamide, but they are not suitable for high-temperature reservoirs $\left(>90^{\circ} \mathrm{C}\right)$ with high-density brine fluids. The functional amide group in polyacrylamide is hydrolyzed at $80-90^{\circ} \mathrm{C}$ [4]. The polymer solution must be stable in harsh conditions of salinity and temperature. Acrylamide-based polymers suffer from some deficiencies such as their rapid hydrolysis, facile thermal degradation, and undesirable properties. To overcome these problems, some vinyl copolymers containing 2-acrylamido-2-methyl-1propane sulfonic acid (AMPS) have been developed. Copolymers of acrylamide with sulfonated comono-mers, especially AMPS, offer hydrogen bonding capability and polyelectrolyte 
behaviour in aqueous solution [5]. Therefore, polymers containing sulfonate groups are expected to offer higher stability in solution [6] and can be tolerant to the action of high salt. Candidate AM-based copolymers suitable for testing can be prepared by copolymerization of AM with another vinyl-type monomers or by the chemical modification of polyacrylamide itself [7].

The aim of this study was to synthesize water soluble acrylmide-based anionic copolymers and to investigate the performance of copolymers with respect to shear resistance and molecular weight from industry application standpoint.

\section{Experimental}

2.1. Materials. Acrylamide (AM) and potassium persulfate were purchased from Merck Co. 2-Acrylamido-2-methyl-1propane sulfonic acid (AMPS) was provided from Aldrich Co. All chemicals were reagent grades and used as received without further purification.

2.2. Copolymerization. Copolymers of AM and AMPS were synthesized via conventional free-radical polymerization. The total monomer concentration was kept constant in all polymerizations at $0.58 \mathrm{~mol}$. Potassium persulfate was used as the initiator. The initiator-to-monomer ratio was $0.1 \mathrm{~mol} \%$. The feed ratio of AM to AMPS was varied from $90: 10,70: 30$, and 50:50 to $30: 70$. The following procedure is a typical polymerization method for AM/AMPS $90: 10$.

The polymerization was carried out in a $1 \mathrm{~L}$ four-necked glass reactor, equipped with a reflux condenser, a mechanical stirrer and a nitrogen inlet gas. Initially the reactor was charged with monomers in degassed and distilled water in definite ratio to obtain a $10 \mathrm{wt} \%$ aqueous solution. The reactor was placed in an oil bath of $25^{\circ} \mathrm{C}$ temperature. The solution was sparged with $\mathrm{N}_{2}$ for $20 \mathrm{~min}$. while stirring. The $\mathrm{pH}$ of reaction solution was adjusted to $8.5 \pm 0.5$ by addition of $\mathrm{NaOH}$ to ensure neutralization of the AMPS monomer to its sodium salt. The water soluble initiator KPS $(0.1 \% \mathrm{~mol}$ of monomers) was dissolved in $10 \mathrm{~mL}$ water and sparged with $\mathrm{N}_{2}$ for $15 \mathrm{~min}$ and then added to the reactor. The mixture was heated at $60^{\circ} \mathrm{C}$ for $6 \mathrm{~h}$. The final solution was clear and highly viscous. At the end of reaction, the reaction mixture was cooled at room temperature and was diluted to 2-3 times its volume with water, and then stirred gently with a mechanical stirrer until a homogeneous solution was obtained. The product was precipitated into acetone while stirring. The product was a white, cotton-like solid which was dried under vacuum at $40^{\circ} \mathrm{C}$ for $6 \mathrm{~h}$ until reaching a constant weight.

2.3. Characterization. A Bruker Fourier transform infrared (FTIR) spectrophotometer was used. The samples were pressed into $\mathrm{KBr}$ pellets. ${ }^{1} \mathrm{H}$ - and ${ }^{13} \mathrm{C}$-NMR spectra were recorded on a Bruker 400 NMR spectrometer at room temperature, with $\mathrm{D}_{2} \mathrm{O}$ as solvent and tetramethylsilane (TMS) as an internal reference.
2.4. Determination of Anionic Degree of Copolymers. The anionic degree of samples was determined by a back titration method [8] and by ${ }^{13} \mathrm{C}$-NMR spectroscopy. In titration method, a $\mathrm{pH}$ meter equipped with a glass and reference electrode quantified the functional groups with different anionic degrees. A quantity of $0.2 \mathrm{~g}$ of each anionic sample was dissolved in $50 \mathrm{~mL}$ distilled water. The $\mathrm{pH}$ of the aqueous solution was about 8.5 . Hydrochloric acid $(0.1 \mathrm{~N})$ solution was dripped until the $\mathrm{pH}$ reached 3.3. The miliequivalent gram of hydrochloric acid consumed was equal to the number of anionic monomer contents of each sample. ${ }^{13} \mathrm{C}-\mathrm{NMR}$ spectroscopy technique was used for determination of the anionic degree of copolymer samples [9].

2.5. Viscosity Measurement in NaCl Solution. Polymer solutions were prepared by dissolving an appropriate amount of vacuum-oven dried purified polymer in $\mathrm{NaCl}$ aqueous solution. The intrinsic viscosity of samples was measured with an Ubbelohde viscometer at $25 \pm 1^{\circ} \mathrm{C}$. The flux time was recorded until reproducible values were obtained. Extrapolation from data obtained for five solution concentrations was used to evaluate the intrinsic viscosity. Apparent viscosities were measured by a Brookfield LVTDV II viscometer. The viscometric experiments were carried out using 20,30, 50, 60, 100 , and 200 shear rates at room temperature. The viscosityaverage molecular weights were calculated by using the MarkHouwink relationship.

\section{Results and Discussion}

Radical copolymerization of AM and AMPS with a variation in monomeric feed ratio was carried out in aqueous solution in order to obtain copolymers containing anionic groups (Scheme 1). Important contributions of each monomer are as follows. Acrylamide (AM) is a hydrophilic and nonionic monomer and has high rate constant for polymerization in water. 2-Acrylamido-2-methyl-1-propane sulfonic acid (AMPS) as the anionic monomer has high reactivity and good hydrolytic stability due to the geminal dimethyl groups and ionization even at low $\mathrm{pH}$ values. The different copolymers were synthesized by varying the feed ratio of monomers (Table 1).

\subsection{Characterization of Copolymer Structure}

3.1.1. Fourier Transform Infrared Spectroscopy. A typical presentation of FTIR spectra of the AM-co-AMPS with $(90: 10)$ molar ratio is shown in Figure 1. The FTIR spectrum of copolymers shows stretching vibration peaks at $3432 \mathrm{~cm}^{-1}$ and $1647 \mathrm{~cm}^{-1}$ for amino and amidic carbonyl groups, respectively. The peaks situated around $1039 \mathrm{~cm}^{-1}$ and $1183 \mathrm{~cm}^{-1}$ are the characteristic peaks of the asymmetric and symmetric bands of sulfonate groups in AMPS unit [10]. It should be mentioned that the effectiveness of monomer reactions can be confirmed because of the disappearance of vinylic absorbance peak at about $1600 \mathrm{~cm}^{-1}$. 
TABLE 1: The monomer feed ratio, the anionic degree, and molecular weight of copolymers.

\begin{tabular}{|c|c|c|c|c|c|}
\hline \multirow{2}{*}{ Sample number } & \multicolumn{2}{|c|}{ Monomer feed ratio } & \multicolumn{2}{|c|}{ Anionic degree (\%) } & \multirow{2}{*}{$M_{w} \times 10^{6}(\mathrm{~g} / \mathrm{mol})$} \\
\hline & $\mathrm{AM}$ & AMPS & Titration method & ${ }^{13} \mathrm{C}-\mathrm{NMR}$ & \\
\hline 1 & 90 & 10 & 6.7 & 6.1 & 8.557449 \\
\hline 2 & 70 & 30 & 27.5 & 26.11 & 6.000686 \\
\hline 3 & 50 & 50 & 47 & 46.9 & 5.224714 \\
\hline 4 & 30 & 70 & 67.3 & 67 & 2.243815 \\
\hline
\end{tabular}

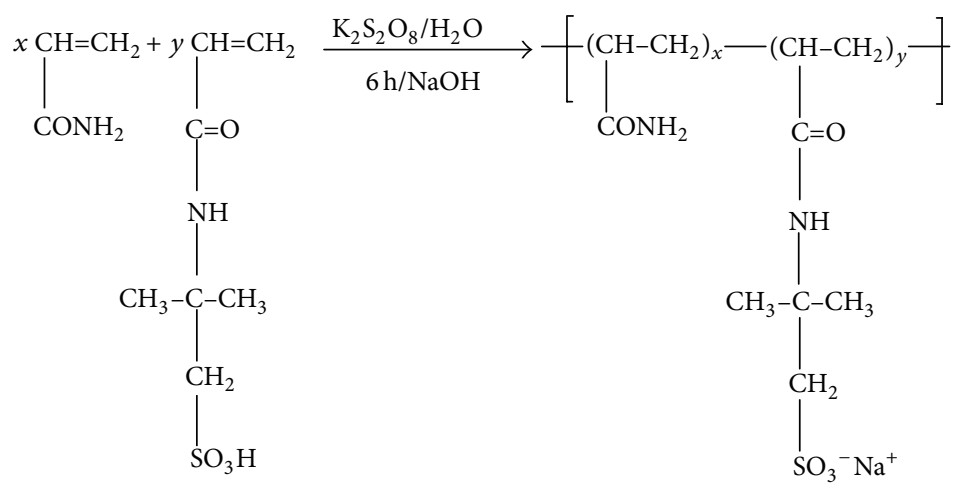

SCHEME 1: Radical copolymerization of acrylamide and 2-acrylamido-2-methyl-1-propane sulfonic acid.

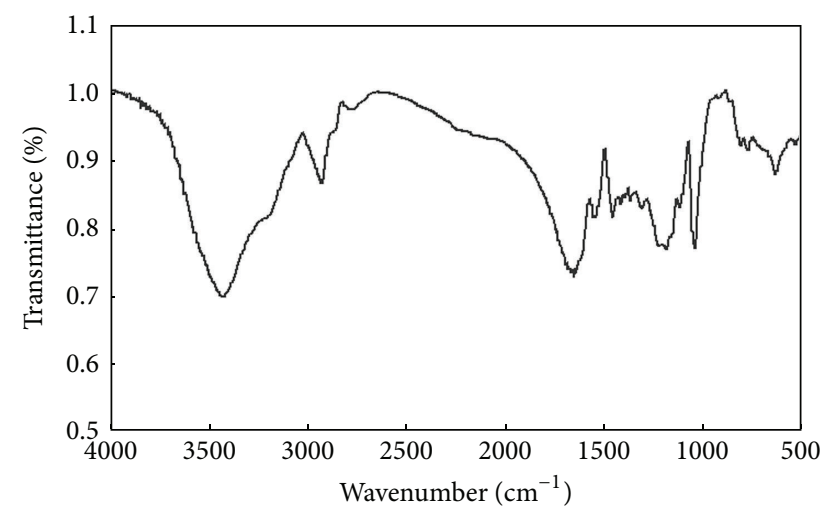

FIGURE 1: FTIR spectra of copolymer AM/AMPS $90: 10$.

3.1.2. Nuclear Magnetic Resonance Spectroscopy. Further evidence for inclusion of two monomers was obtained by the ${ }^{1} \mathrm{H}-\mathrm{NMR}$ and ${ }^{13} \mathrm{C}-\mathrm{NMR}$ spectra of the copolymer. A typical presentation of the ${ }^{1} \mathrm{H}-\mathrm{NMR}$ and ${ }^{13} \mathrm{C}-\mathrm{NMR}$ spectra of AM-co-AMPS copolymers is shown in Figures 2 and 3, respectively. The ${ }^{1} \mathrm{H}-\mathrm{NMR}$ spectra show the disappearance of olefinic protons and the signals corresponding to the characteristic protons coming from both monomers. In the ${ }^{1} \mathrm{H}-\mathrm{NMR}$ spectra, the peak with chemical deviation of 1.29$1.55 \mathrm{ppm}$ corresponds to the hydrogens in the main chain. The hydrogen atoms of $\mathrm{CH}_{3}$ groups are detected at $2.03 \mathrm{ppm}$. The hydrogens of the $\mathrm{CH}_{2}$ group bonded to $\mathrm{SO}_{3} \mathrm{Na}$ are detected at $3.2 \mathrm{ppm}$. The signal at $4.6 \mathrm{ppm}$ corresponds to the hydrogens of the $\mathrm{NH}$ and $\mathrm{NH}_{2}$ groups and the water in the solvent $\mathrm{D}_{2} \mathrm{O}$ (99.9\%) and in the copolymer [11].

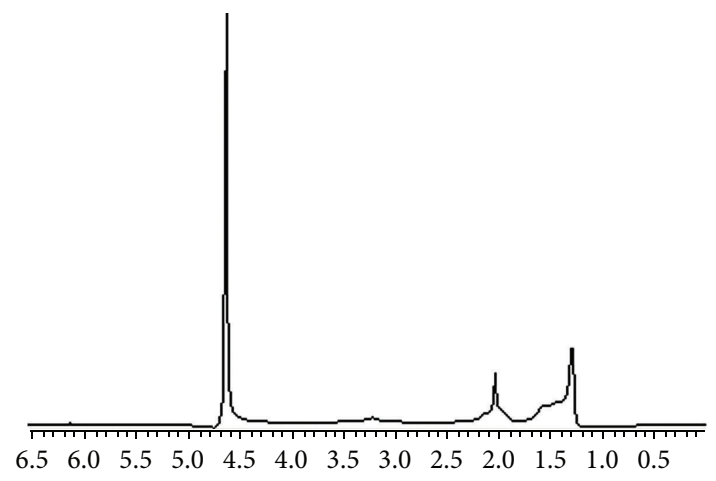

FIgURE 2: ${ }^{1} \mathrm{H}-\mathrm{NMR}$ spectrum of copolymer AM/AMPS $90: 10$.

${ }^{13} \mathrm{C}-\mathrm{NMR}$ typical copolymer: AM C=O $180.33 \mathrm{ppm}$; NaAMPS C=O 176.57 ppm; chain $\mathrm{CH} 42.6$ ppm; chain $\mathrm{CH}_{2}$ 35.6 ppm; $\mathrm{CH}_{3}$ (AMPS) $27.2 \mathrm{ppm} ; \mathrm{CH}_{2}-\mathrm{SO}_{3}{ }^{-} 58.22 \mathrm{ppm} ; \mathrm{CH}$ (AMPS) $53.04 \mathrm{ppm}$ [11]. As expected, the ${ }^{1} \mathrm{H}-\mathrm{NMR}$ and ${ }^{13} \mathrm{C}$ NMR confirmed the presence of both monomers in the copolymers.

3.2. Determination of Anionic Degree of Copolymers. The number of anionic monomer content of each sample is known as the anionic degree. The anionic degree obtained from ${ }^{13} \mathrm{C}$-NMR peak integration and titration methods are given in Table 1 . The ${ }^{13} \mathrm{C}-\mathrm{NMR}$ analysis was carried out for calculation of copolymer composition using the well-resolved carbonyl peaks due to different chemical shifts of AM and AMPS functional carbonyl groups. 


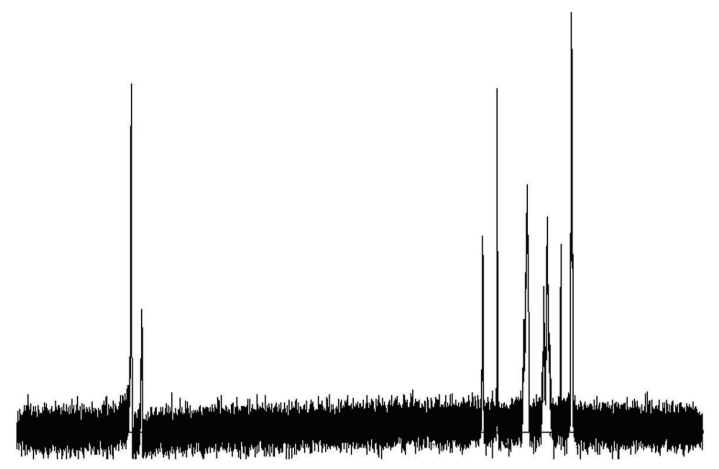

FIgURE $3:{ }^{13}$ C-NMR spectum of copolymer AM/AMPS $90: 10$.
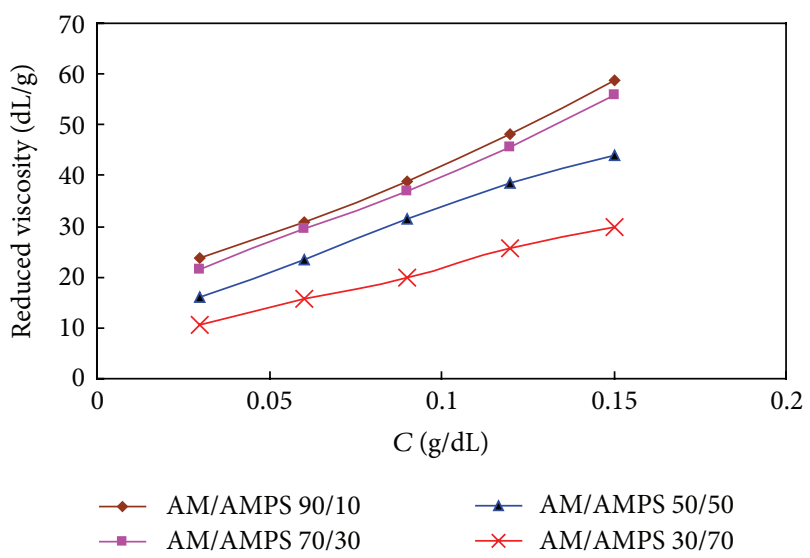

FIGURE 4: Variation of reduced viscosity of copolymer solution versus copolymer concentration in aqueous salt solution at $25^{\circ} \mathrm{C}$.

The weight fraction of AMPS in the copolymer was calculated according to the following equation [9]:

$$
\operatorname{mol} \% \mathrm{AMPS}=\frac{I_{\mathrm{AMPS}}}{I_{\mathrm{AMPS}}+I_{\mathrm{AM}}} \times 100 .
$$

Figure 3 represents the ${ }^{13} \mathrm{C}-\mathrm{NMR}$ spectrum of AM/AMPS copolymer $(90: 10)$. Copolymer compositions were determined using amide carbonyl peaks. $I_{\mathrm{AMPS}}$ and $I_{\mathrm{AM}}$ represent the integral area of carbonyl peak of NaAMPS and AM, respectively. Comparisons of these values with those of titration method are shown in Table 1. The results showed a relatively good agreement between the data obtained by the two methods.

3.3. Viscosity Measurement in $\mathrm{NaCl}$ Solution. Due to electrostatic interactions between the negatively charged groups along the chain, the polyelectrolyte solutions show quite different behaviors compared with neutral polymers. Thus, it is well known that in the case of pure solutions of ionic polymer, the $\eta_{\mathrm{sp}} / \mathrm{C}-\mathrm{C}$ curves show a maximum. These $\eta_{\mathrm{sp}} / \mathrm{C}-\mathrm{C}$ curves cannot be extrapolated to zero polyelectrolyte concentration for intrinsic viscosity determination. This problem can be solved by means of some equations such as Fedors' equation modified by Rao [12] or by addition of low molecular weight salts [13] which have charge screening effect. In present

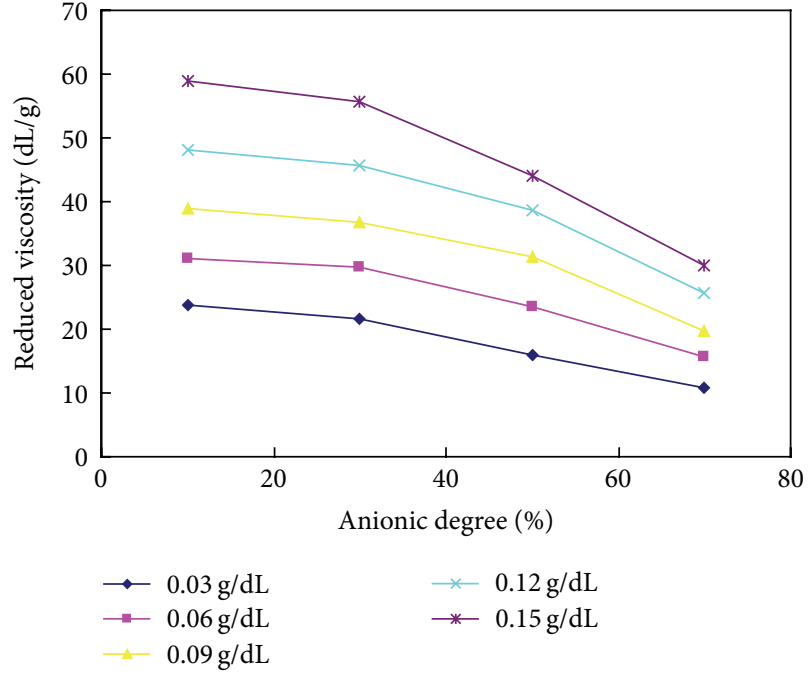

FIGURE 5: Variation of reduced viscosity versus anionic charge at various copolymer concentrations.

study, the second method was used to eliminate the repulsive interactions of charges between the different segments of the copolymer. Therefore, intrinsic viscosity is evaluated by extrapolation method using aqueous copolymer solution in $\mathrm{NaCl}$ at $25^{\circ} \mathrm{C}$ as also reported by other researchers. The $\mathrm{NaCl}$ concentration sufficiently high for the polymer to present a neutral polymer behavior was determined to be $0.5 \mathrm{M}$.

The variation of the reduced viscosity, $\eta_{\mathrm{Sp}} / \mathrm{C}$, versus the concentration of copolymers in aqueous salt solution is presented in Figure 4. It is observed that the reduced viscosity increases with increasing polymer concentration.

\subsection{The Effect of Anionic Charge of Copolymer on Reduced} Viscosity and Molecular Weight. The effect of anionic degree on reduced viscosity and molecular weight were evaluated and studied. The reduced viscosity as a function of the anionic degree of copolymers is shown in Figure 5. A decrease in reduced viscosity is observed with an increase of negative charges along the copolymer chain at various concentrations of copolymer. It can be seen that in the presence of salt, the macroions shrank and became more like spheres which ultimately results in a coil-like configuration. Therefore, the hydrodynamic volume of the chains is reduced which leads to lower reduced viscosity $[8,14]$. The viscosity-average molecular weights of copolymers could be calculated by using the Mark-Houwink relationship [15]:

$$
M_{w}=\left\{10,000 \times \frac{[\eta]}{3.73}\right\}^{1 / 0.66} .
$$

The viscosity-average molecular weights in Table 1 seem to confirm samples of having high molecular weights. In this case, the effect of anionic degrees in copolymer chains on molecular weight was investigated. According to the results, the $M_{w}$ of copolymers decrease with an increase in the weight percent of AMPS in the copolymerization feed. The reduced viscosity is directly proportional with the molecular 
TABLE 2: Viscosities of samples at different ranges of shear rates.

\begin{tabular}{|c|c|c|c|c|c|c|c|}
\hline \multirow[b]{2}{*}{ Sample number } & \multirow[b]{2}{*}{ AMPS (\%) } & \multicolumn{6}{|c|}{ Shear rate (rpm) } \\
\hline & & 20 & 30 & 50 & 60 & 100 & 200 \\
\hline & & \multicolumn{6}{|c|}{ Viscosity $(c \mathrm{P})$} \\
\hline 1 & 10 & 452 & 398 & 332 & 310 & 252 & 150 \\
\hline 2 & 30 & 259 & 248 & 230 & 223 & 203 & 160 \\
\hline 3 & 50 & 160 & 156 & 150 & 148 & 143 & 131 \\
\hline 4 & 70 & 85 & 84 & 80 & 78 & 74 & 70 \\
\hline
\end{tabular}

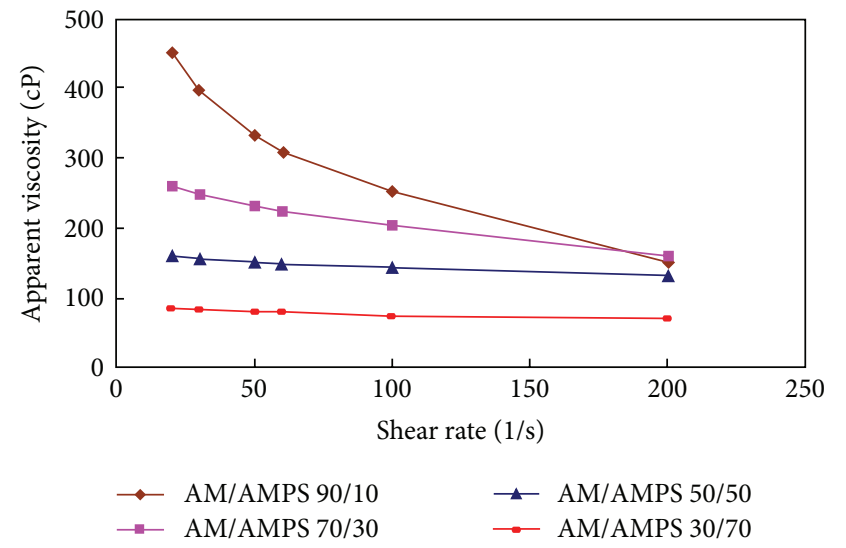

Figure 6: Shear rate dependence of apparent viscosity of a $0.5 \%$ $(\mathrm{w} / \mathrm{w})$ aqueous solution of copolymers at $25^{\circ} \mathrm{C}$.

weight, so it can be concluded that the reduction trend of both, the $M_{w}$ and $\eta_{\mathrm{sp}} / \mathrm{C}$ with respect to anionic charges is expected. This result is probably due to repulsive effects in AMPS monomer which are inherent to the anionic polymers. Therefore, as the copolymerization reaction proceeds from $10 \%$ to $70 \%$ weight percent of negative charges, the rate slows down due to accumulation of negative charges and the molecular weight of copolymers decreases with increasing the anionic degrees of copolymers. It should be mentioned that the repulsion forces between the macroradicals and entering monomers is probably responsible for the reduction of the chain growth rate constant which leads in low molecular weight copolymers [16].

\subsection{Rheological Behavior}

3.5.1. Effect of Shear Rate on Solution Viscosity. The viscosities of samples subjected to different shear rates were evaluated. It is apparent from Figure 6 that the copolymer solutions display non-newtonian shear thinning behavior within the experimental range of shear rates (Table 2). The curves show that the apparent viscosity $\left(\eta_{\text {app }}\right)$ decreases with increasing the shear rate of the solutions. A reduction in viscosity as shear rate increases is an indication of possible electrostatic interactions between the particles surpassed by viscous forces applied to the solutions. As a result, at high shear rate the polymer chains are aligned in the direction of flow which results in a viscosity reduction or shear-thinning behaviour.
Also, a decrease in apparent viscosity is observed with increased negative charges along the polymer chain; in the presence of salt, the macroions shrank and became more like spheres which ultimately results in a coil-like configuration [8]. This helps in resisting the deformation of the structure on shearing, leading to the reduction in viscosity. As expected, the hydrodynamic volume of the chains is reduced which leads to lower apparent viscosity. In addition, from a rheological point of view, the presence of some hydrophobic groups such as $\mathrm{CH}_{3}$, along with hydrophilic groups, may help with shear resistance [5].

It can be observed from Figure 6 that a AM/AMPS copolymer ratio of 90:10 was more shear-sensitive than other copolymer ratios. In fact, the copolymers of AM/AMPS $(30: 70)$ and AM/AMPS $(50: 50)$ with higher anionic charges were more shear-resistant than the other copolymers. This order may also support the contribution of the hydrophilic group to shear resistance. Also, the stronger hydrogen bonding ability of the $\mathrm{SO}_{3}{ }^{-}$group should have helped with the reformation of the structures in copolymers during shearing [5].

\section{Conclusions}

Water soluble acrylmide-based anionic copolymers were prepared by free radical solution polymerization. By changing the AMPS monomer feed ratio from 10 to 70 weight percent, while keeping other reaction conditions constant, different anionic copolymers were synthesized. The chemical structure of copolymers was evaluated and confirmed with ${ }^{1} \mathrm{H}-\mathrm{NMR}$, ${ }^{13} \mathrm{C}-\mathrm{NMR}$, and FTIR spectroscopic methods. The anionic degree of copolymers was determined from ${ }^{13} \mathrm{C}$-NMR peak integration and titration method. The data obtained by these two methods seem to be in good agreement. The measured molecular weight of samples shows that we have reached a high molecular weight with excellent solubility. According to the results, the copolymer solutions show a nonnewtonian shear thinning behaviour. At high shear rate, the polymer chains are aligned in the direction of flow which result in reduced viscosity or shear-thinning behaviour. An assessment of the anionic charge on reduced viscosity and molecular weight showed decrease in reduced viscosity and $M_{w}$ would occur with an increase in weight portion of AMPS monomer in the feed. This effect may be attributed to the repulsive effects in the AMPS monomer which is an inherent property in anionic polymers. It should be mentioned that AM/AMPS (50:50) samples have the best performance with 
respect to shear resistance and required high molecular weight. These properties may make this polymer of potential technological interest in oil recovery and other important commercial applications.

\section{Conflict of Interests}

The authors declare that there is no conflict of interests regarding the publication of this paper.

\section{Acknowledgment}

The Authors would like to thank Miss Houri Mivehchi, Editor of Iranian Polymer Journal, for editing this paper.

\section{References}

[1] C. A. Finch, Industrial Water Soluble Polymers, The Royal Society of Chemistry, Cambridge, UK, 1996.

[2] S. E. Morgan and C. L. Mccormick, "Water-soluble polymers in enhanced oil recovery," Progress in Polymer Science, vol. 15, no. 1, pp. 103-145, 1990.

[3] A. Rabiee, "Acrylamide-based anionic polyelectrolytes and their applications: a survey," Journal of Vinyl and Additive Technology, vol. 16, no. 2, pp. 111-119, 2010.

[4] A. Sabhapondit, A. Borthakur, and I. Haque, "Characterization of acrylamide polymers for enhanced oil recovery," Journal of Applied Polymer Science, vol. 87, no. 12, pp. 1869-1878, 2003.

[5] N. Garoff, N. Nilvebrant, and C. Fellers, "Rheological behavior of N,N-dimethyl acrylamide-acrylamido methylpropane sulphonate copolymer," Journal of Applied Polymer Science, vol. 85, no. 7, pp. 1521-1529, 2002.

[6] B. Peng, S. Peng, B. Long, Y. Miao, and W. Guo, "Properties of high-temperature-resistant drilling fluids incorporating acrylamide/(acrylic acid)/(2-acrylamido-2-methyl-1-propane sulfonic acid) terpolymer and aluminum citrate as filtration control agents," Journal of Vinyl and Additive Technology, vol. 16, no. 1, pp. 84-89, 2010.

[7] M. B. Hocking, K. A. Klimchuk, and S. Lowen, "Water-soluble acrylamide copolymers. VIII. Preparation and characterization of polyacrylamide-co-N-t-butylacrylamide," Journal of Polymer Science A: Polymer Chemistry, vol. 39, no. 12, pp. 1960-1977, 2001.

[8] A. Rabiee, M. E. Zeynali, and H. Baharvand, "Synthesis of high molecular weight partially hydrolyzed polyacrylamide and investigation on its properties," Iranian Polymer Journal, vol. 14, no. 7, pp. 603-608, 2005.

[9] B. H. Hutchinson and C. L. McCormick, "Water-soluble copolymers: 15 . Studies of random copolymers of acrylamide with $\mathrm{N}$ substituted acrylamides by 13 C n.m.r." Polymer, vol. 27, no. 4, pp. 623-626, 1986.

[10] F. Rosa, J. Bordado, and M. Casquilho, "Hydrosoluble copolymers of acrylamide-(2-acrylamido-2-methylpropanesulfonic acid). Synthesis and characterization by spectroscopy and viscometry," Journal of Applied Polymer Science, vol. 87, no. 2, pp. 192-198, 2003.

[11] J. Travas-Sejdic and A. Easteal, "Study of free-radical copolymerization of acrylamide with 2-acrylamido-2-methyl1-propane sulfonic acid," Journal of Applied Polymer Science, vol. 75, pp. 619-628, 2000.
[12] S. Dragan, M. Mihai, and L. Ghimici, "Viscometric study of poly(sodium 2-acrylamido-2-methylpropanesulfonate) and two random copolymers," European Polymer Journal, vol. 39, no. 9, pp. 1847-1854, 2003.

[13] L. Ghimici and F. Popescu, "Determination of intrinsic viscosity for some cationic polyelectrolytes by Fedors method," European Polymer Journal, vol. 34, no. 1, pp. 13-16, 1998.

[14] M. E. Zeynali, A. Rabii, and H. Baharvand, "Synthesis of partially hydrolyzed polyacrylamide and investigation of solution properties (viscosity behaviour)," Iranian Polymer Journal, vol. 13, no. 6, pp. 479-484, 2004.

[15] Y. M. Wu, Y. P. Wang, Y. Q. Yu, J. Xu, and Q. F. Chen, "Dispersion polymerization of acrylamide with 2-acrylamido-2-methyl-1propane sulfonate in aqueous solution," Journal of Applied Polymer Science, vol. 102, no. 3, pp. 2379-2385, 2006.

[16] J. C. Salamone, Polymeric Materials Encyclopedia, vol. 1, CRC, New York, NY, USA, 1996. 

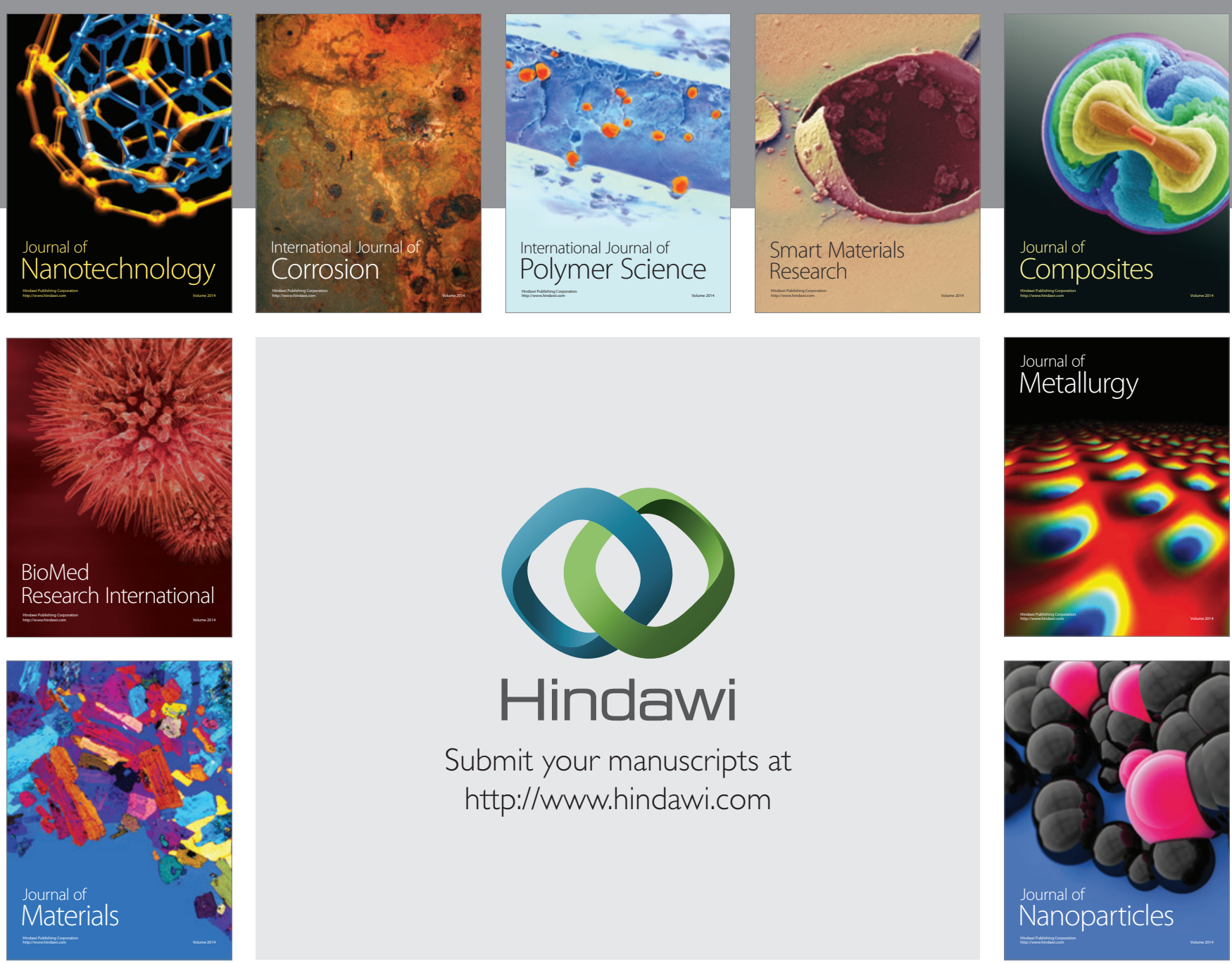

Submit your manuscripts at http://www.hindawi.com
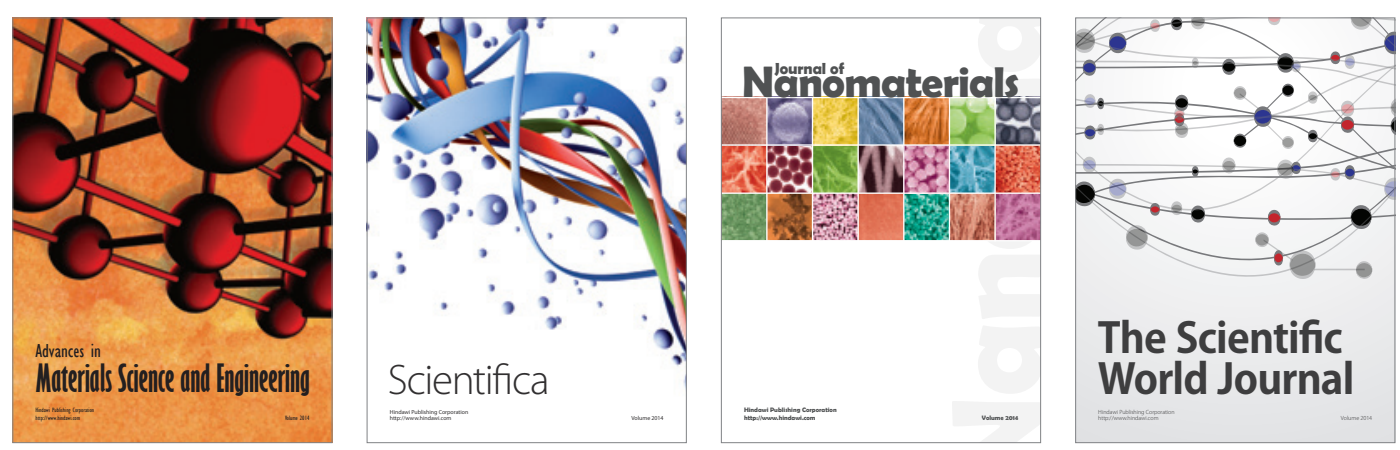

\section{The Scientific World Journal}
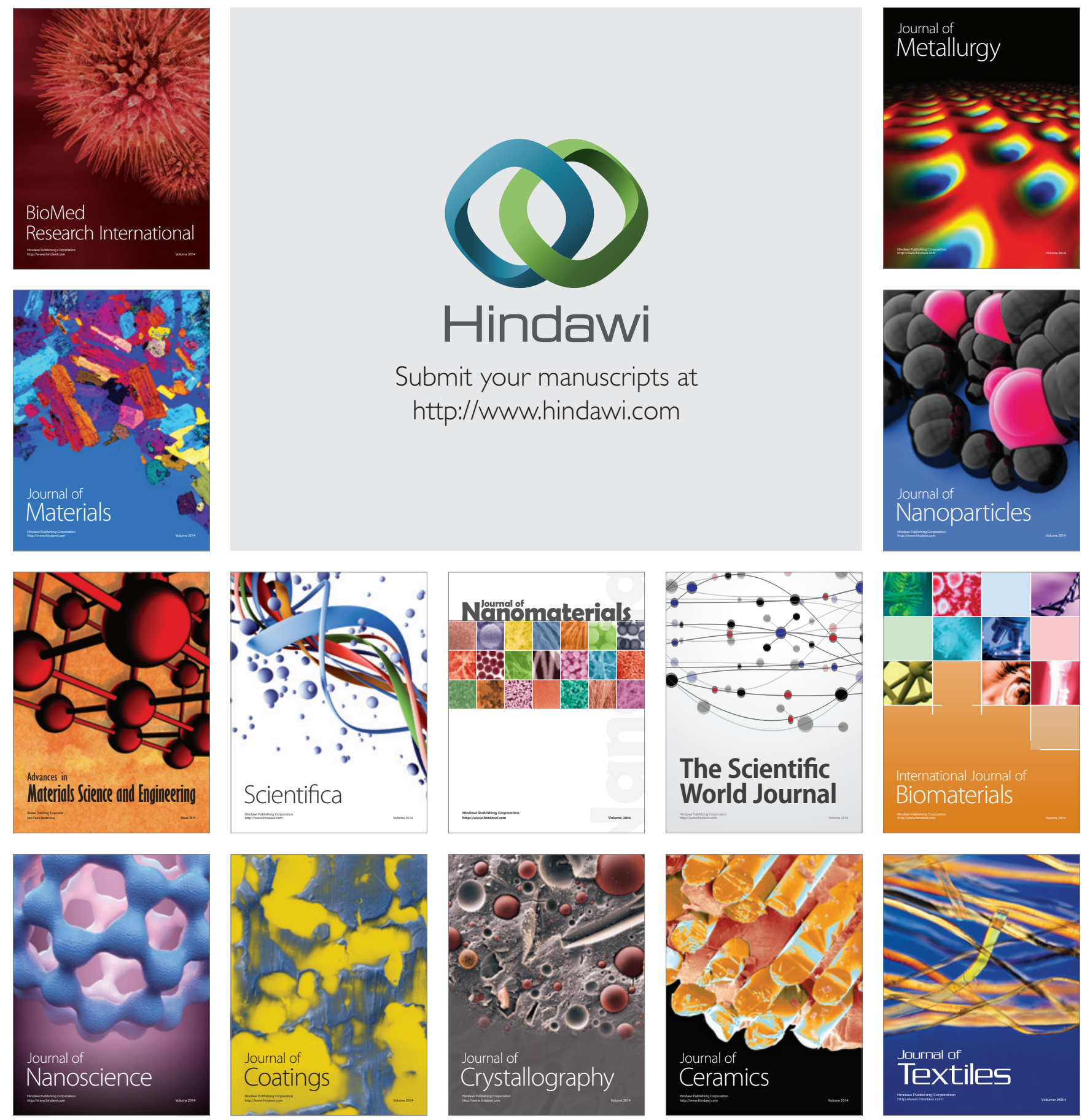